\title{
EcoIMU: A Dual Triaxial-Accelerometer Inertial Measurement Unit for Wearable Applications
}

\author{
Yi-Lung Tsai, Ting-Ting Tu \\ ${ }^{1}$ Department of Computer Science \\ National Tsing Hua University \\ Hsinchu, Taiwan 30013 \\ \{iverlon, corila.tu\}@gmail.com
}

\author{
Hyeoungho Bae, Pai H. Chou ${ }^{1,2}$ \\ ${ }^{2}$ Center for Embedded Computer Systems \\ University of California, Irvine \\ Irvine, CA 92697-2625 USA \\ \{hyeoungb,phchou\}@uci.edu
}

\begin{abstract}
This paper describes EcoIMU, a gyro-free, wearable inertial measurement unit (IMU) built with a pair of triaxial accelerometers that are spatially separated and wirelessly connected on the body. It can output the translation and rotation of the body for the purpose of motion tracking and dead reckoning applications. It mitigates error accumulation and drift problems using domain knowledge including pause identification and geometric constraints on the two nodes. Experimental results show EcoIMU to have less drift error while consuming significantly lower power than comparable IMUs built with MEMS gyroscopes and accelerometers.
\end{abstract}

Keywords-Inertial Measurement Unit; gyro-free IMU; motion tracking; accelerometer; body sensor

\section{INTRODUCTION}

Motion measurement is a fundamental function for many sensing applications. In 3D space, an object has six degrees of freedom: translation (linear motion) along and rotation about each of the $\mathrm{X}, \mathrm{Y}, \mathrm{Z}$ axes. One can measure the motion of the object either by external observation or in-situ motion sensing. External observation includes video, sound, and radar, which may be effective for determining the absolute position of an object, but they may suffer from obstruction and limited resolution, which must be overcome by installing sensors from many different viewing angles. In this paper, we consider insitu motion sensors, called inertial measurement units (IMU).

An IMU is a system for measuring motion of an object in free space relative to an inertial frame. Sensors for inertial measurement consist primarily of accelerometers and gyroscopes.

\section{A. Single Triaxial Accelerometer}

A triaxial accelerometer alone can measure three degrees of freedom. If an object can be assumed to have either zero translation or zero rotation, then a single triaxial accelerometer suffices for measuring either rotation or translation, respectively. On earth, gravity of $1 \mathrm{~g}$ is always present. By assuming rotation without translation, a triaxial accelerometer can be used to measure tilt by computing the orientation of this 1g gravity vector. With some domain-specific knowledge, it may be possible to re-orient the sensor by assuming that the subject holds static postures (i.e., zero translation and zero rotation) from time to time. If the sensing data shows a constant acceleration of $1 \mathrm{~g}$ in a fixed orientation for a certain amount of time, then one may guess that the subject is holding a static posture.

\section{B. Gyroscopes}

To distinguish between rotation and translation, gyroscopes are often used. A traditional gyroscope, or gyro for short, consists of a rotor that spins freely to maintain angular momentum and therefore its original orientation. Vibrating structure gyroscopes replace rotors with proof masses that vibrate, which also has the effect of maintaining the original orientation but can be made smaller and cheaper with MEMS technology. Deviation from the original orientation can be measured to derive the rotation in terms of angle and angular velocity. After canceling the projection of rotation onto the three axes, the remaining linear acceleration can then be interpreted as translation. However, gyroscopes also have their issues. Each gyroscope is limited by a maximum angular velocity that can be tolerated before the rotor or vibrating structure's original orientation is changed. Another issue is the relatively high power consumption compared to accelerometers, even in MEMS implementations.

\section{Gyro-Free, Accelerometers-only IMU}

It is possible to construct gyro-free IMUs using accelerometers only, although this is not the only way to build a gyro-free IMU (GF-IMU). Several wireless sensor platforms, including the ultra-compact Eco platform that we use, have an onboard triaxial accelerometer. It is possibly the most practical, as it requires no additional sensor module, can be deployed as a sensing network that is physically distributed, loosely coupled, and tightly collaborative. The paper targets primarily this architecture. EcoIMU is the first practical implementation of an IMU of this class. Unlike other models that rely on six uniaxial accelerometers, we use a pair of triaxial ones. By reducing the number of accelerometer components and circuit boards, we make it more convenient to wear and deploy. This IMU can be more accurate than MEMS gyro-based IMUs, as shown in a number of test cases.

\section{BACKGROUND AND RELATED WORK}

Gyro-free IMU has been under research for over 20 years [1]. Although it was known in theory that a minimum of 

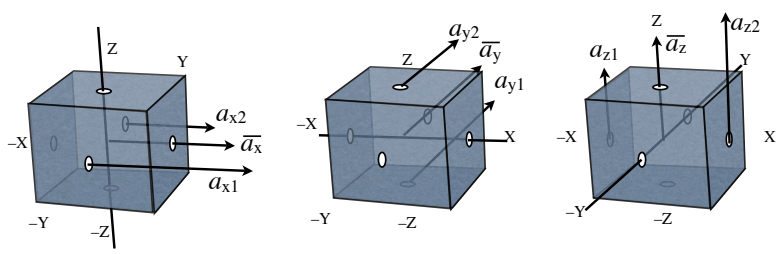

Fig. 1. Bounding cube

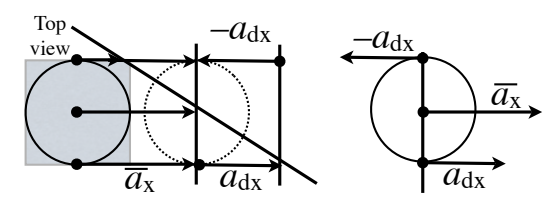

Fig. 2. Decomposed vectors for translation (as average) and rotation (as half of difference) based on acceleration values measured on two parallel planes. $\mathrm{X}$ accelerations on $\mathrm{XY}$ plane shown, rotating about $\mathrm{Z}$ axis (towards the reader). This picture can be generalized to $\mathrm{Y}$ acceleration on $\mathrm{YZ}$ plane and $\mathrm{Z}$ acceleration on $\mathrm{ZX}$ plane.

six uniaxial accelerometers are required for a complete GFIMU system, it was not realized until Chen et al proposed a basic cube model [2]. Later, more math models [3]-[5] were proposed to improve the accuracy based on the cube model.

Both gyro- and gyro-free IMUs have been published, but there have been no real practical implementations of GF-IMUs in body sensor networks. The issues that must be addressed include how to take time-synchronized samples, make them small and wearable, use as few devices as possible, and be adaptive to a variety of geometries. In the rest of this section, we review the basic kinematics for the purpose of building up an accelerometer-based GF-IMU.

Linear acceleration in the three dimensions are the average of the pair of acceleration values measured at the center of parallel surfaces of a bounding cube, as shown in Fig. 1:

$$
\vec{a}=\left(a_{x}, a_{y}, a_{z}\right)=\left(\frac{a_{x 1}+a_{x 2}}{2}, \frac{a_{y 1}+a_{y 2}}{2}, \frac{a_{z 1}+a_{z 2}}{2}\right)
$$

This is because the center point by definition experiences zero contribution to measured linear acceleration from rotation. The average of the two therefore is the linear acceleration. This is shown in Fig. 2.

For rotation, the two sides rotate in opposite directions at the same rate.

$$
\begin{array}{lll}
a_{x 1}=\bar{a}_{x}+a_{d x} & a_{y 1}=\bar{a}_{y}+a_{d y} & a_{z 2}=\bar{a}_{z}-a_{d z} \\
a_{x 2}=\bar{a}_{x}-a_{d x} & a_{y 2}=\bar{a}_{y}-a_{d y} & a_{z 1}=\bar{a}_{z}+a_{d z}
\end{array}
$$

Therefore, the accelerations due to rotation in the $\mathrm{X}, \mathrm{Y}, \mathrm{Z}$ directions are

$$
a_{d x}=\frac{a_{x 2}-a_{x 1}}{2} \quad a_{d y}=\frac{a_{y 2}-a_{y 1}}{2} \quad a_{d z}=\frac{a_{z 2}-a_{z 1}}{2}
$$

However, these are linear accelerations due to rotation, rather than angular acceleration. To recall, angular acceleration is normalized to unit radius, and the radius here is $1 / 2$ the separation between the pair of accelerometers on the parallel

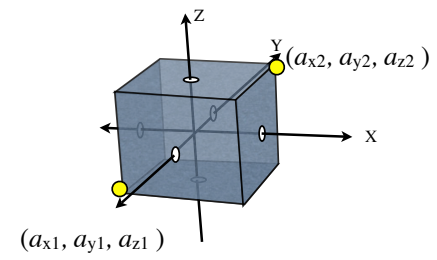

Fig. 3. Two triaxial accelerometers on opposite corners of a cube.

planes. Therefore, we can find $\vec{\alpha}_{z x y}$ and derive $\vec{\omega}_{z x y}, \vec{\theta}_{z x y}$ as follows: We denote angular acceleration along $\mathrm{Z}, \mathrm{X}, \mathrm{Y}$ axes as $\alpha_{z}, \alpha_{x}, \alpha_{y}$, on XY, YZ, ZX planes, respectively.

$$
\begin{aligned}
\vec{\alpha}_{z x y} & =\left(\frac{a_{d x}}{r}, \frac{a_{d y}}{r}, \frac{a_{d z}}{r}\right) \\
\vec{\omega}_{z x y}(t) & =\vec{\omega}_{z x y}(t-\Delta t)+\Delta t \vec{\alpha}_{z x y}(t) \\
\vec{\theta}_{z x y}(t) & =\vec{\theta}_{z x y}(t-\Delta t)+\Delta t \vec{\omega}_{z x y}(t)
\end{aligned}
$$

where $r$ is $1 / 2$ the distance between the two accelerometers on parallel surfaces.

\section{Problem Statement}

Triaxial accelerometers are widely available often in the same-sized packages as uniaxial ones. It would be very attractive if we can deploy a pair of triaxial accelerometers instead of six uniaxial ones. The problem statement here is to derive an IMU based on a pair of triaxial accelerometers by projecting these sensing axis onto the cube model with six uniaxial ones as described in the previous section. More specifically, we propose to locate the two triaxial accelerometers on opposite corners of the bounding cube.

Fig. 3 shows the deployment positions. The math is a variation of the formulation above. For translation, $\left(\bar{a}_{x}, \bar{a}_{y}, \bar{a}_{z}\right)$ are the average of the respective pair, and the equations are the same as before. That is, the center point experiences no rotation-contributed linear acceleration and thus must be all translation, whereas the two sampling points rotate by linearly accelerating in opposite directions: as before:

$$
\vec{a}=\left(\frac{a_{x 1}+a_{x 2}}{2}, \frac{a_{y 1}+a_{y 2}}{2}, \frac{a_{z 1}+a_{z 2}}{2}\right) .
$$

Note that these equations hold even though the two triaxial accelerometers are deployed at different parts of the cube from the six uniaxial case. To prove this, consider the case of $\mathrm{X}$ axis. The two $\mathrm{X}$ sensors are on opposite corners of a cube and measure acceleration in the same direction as the $\mathrm{X}$ axis. Their average is exactly the acceleration of the center of the cube in the same direction. For rotation, let us consider the case of the two $\mathrm{X}$ acceleration sensors on opposite corners of a cube, and the measured acceleration values are $\left(a_{x 1}, a_{x 2}\right)$, respectively. This is shown in Fig. 4. Assume there is a cylinder that bounds the cube along the $\mathrm{Z}$ axis (on $\mathrm{XY}$ plane), the diameters of the three bounding cylinders are

$$
\vec{d}_{z x y}=\left(\sqrt{L_{x}^{2}+L_{y}^{2}}, \sqrt{L_{y}^{2}+L_{z}^{2}}, \sqrt{L_{z}^{2}+L_{x}^{2}}\right)
$$



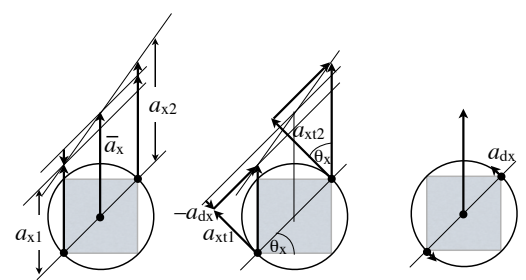

Fig. 4. Top view of enclosing cylinder along $\mathrm{Z}$ axis on two parallel $\mathrm{XY}$ planes. The angular acceleration is derived based on the tangential projection of the $\mathrm{X}$ acceleration values.

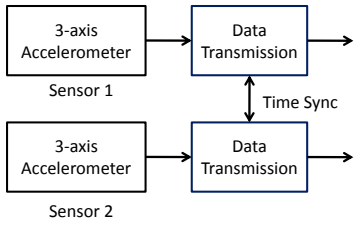

(a) Node Subsystem

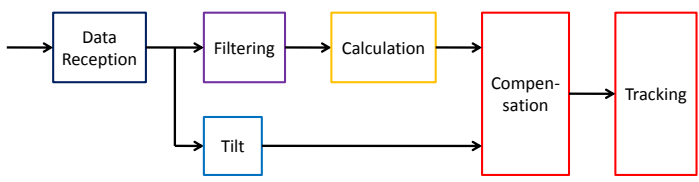

(b) Host Subsystem

Fig. 5. System block diagram for EcoIMU.

on X-Y, Y-Z, and Z-X planes.

The tangential angle of the measured accelerations are

$$
\vec{\theta}_{x, y, z}=\left(\arcsin \left(L_{y} / d_{z}\right), \arcsin \left(L_{z} / d_{x}\right), \arcsin \left(L_{x} / d_{y}\right)\right)
$$

The tangential projections of the acceleration of Eqn. (2) are

$$
\begin{array}{ll}
a_{x t 1}=a_{x 1} \cos \left(\theta_{x}\right) & a_{x t 2}=a_{x 2} \cos \left(\theta_{x}\right) \\
a_{y t 1}=a_{y 1} \cos \left(\theta_{y}\right) & a_{y t 2}=a_{y 2} \cos \left(\theta_{y}\right) \\
a_{z t 1}=a_{z 1} \cos \left(\theta_{z}\right) & a_{z t 2}=a_{z 2} \cos \left(\theta_{z}\right)
\end{array}
$$

From Eqns. (3) and (4), the linear acceleration is $1 / 2$ the difference between the two acceleration values, and the angular acceleration (along Z, X, Y axes respectively) is the linear acceleration normalized to unit radius:

$$
\begin{gathered}
a_{d x}=\frac{a_{x t 2}-a_{x t 1}}{2} \quad a_{d y}=\frac{a_{y t 2}-a_{y t 2}}{2} \quad a_{d z}=\frac{a_{z t 2}-a_{z t 2}}{2} \\
\vec{\alpha}_{z x y}=\left(\frac{2 a_{d x}}{d_{z}}, \frac{2 a_{d y}}{d_{x}}, \frac{2 a_{d z}}{d_{y}}\right) .
\end{gathered}
$$

\section{SySTEM DESIGN AND IMPLEMENTATION}

This section describes the architecture and implementation of EcoIMU. The block diagram is shown in Fig. 5.

\section{A. Architecture and Platform}

The architecture of EcoIMU includes the node subsystem and the host subsystem. The node subsystem consists of multiple wireless sensor nodes that are responsible for sampling time-synchronized acceleration data and transmitting them to

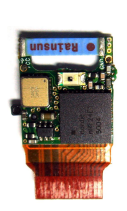

(a)

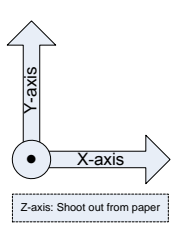

(b)

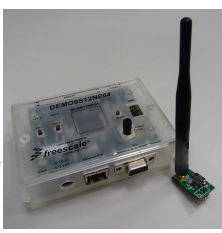

(c)
Fig. 6. Testing environment. (a) Eco. (b) Triaxial directions of Eco's accelerometer. (c) Freescale DEMO9S12NE64 Platform and Nordic nRF24L01 module.

the host. The host subsystem is responsible for computing the IMU functions using the collected data from the accelerometers. The block diagrams of the two subsystems are shown in Fig. 5. The issues to address are time synchronization, sampling, wireless communication protocol, and power management.

We have implemented EcoIMU using an ultra-compact wireless sensor platform, Eco, where the node occupies $1 \mathrm{~cm}^{3}$ in volume and weighs 2 grams including the rechargeable battery and antenna, as shown in Fig. 6(a). Eco contains a Nordic nRF24E1 integrated radio and 8051-based microcontroller, a Hitachi Metals $\mathrm{H} 34 \mathrm{C}$ triaxial accelerometer with $\pm 3 \mathrm{~g}$ range, and an $80 \mathrm{mAh}$ rechargeable lithium polymer battery. The directions of the three axes are shown in Fig. 6(b). The acceleration outputs are sampled by the nRF24E1's builtin 12-bit ADC at 143 samples/sec.

For the host subsystem, we connect a base station board to a PC over Fast Ethernet (100 base/T). As shown in Fig. 6(c), the base station is built by connecting the Freescale DEMO9S12NE64 board to an RF module based on the nRF24L01 transceiver, which is compatible with Eco. All the high-level processes including filtering, velocity and placement calculation, and so on are executed on the PC running a Python script for this prototype. A future version can perform the processing on a wearable board.

\section{B. Data Filtering, Tilt and Compensation}

The raw data from the accelerometers cannot be plugged into the equations without any filtering and resetting. Due to various noises or packet loss, sometimes data can be out of range and thus need to be filtered out, and a relatively simple filter has been implemented for the experiments here.

However, one commonly known problem with accelerometer-based IMUs is error accumulation. That is, noise or quantification error can become significant after double integration. We employ several heuristics to perform recalibration for translation and rotation based on domain knowledge. First, if data from many consecutive packets remain above the threshold value, then it indicates some rather extremes or impossible condition for the wearable application, and it triggers a reset. Second, with the help of gravity, we can compute the angle of tilt. From the tilt with respect to the gravity vector, rotation directions and angles are known and thus aid revising the rotation error. Moreover, as the sensor nodes moving, compensation is made on the fly. If the 


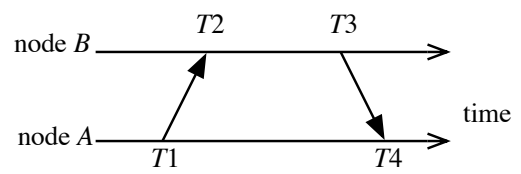

Fig. 7. Time synchronization by two-way message exchange

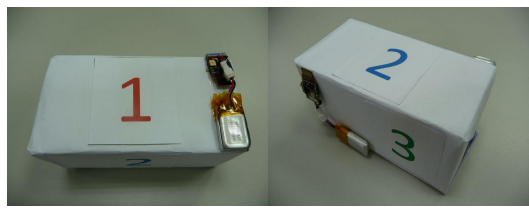

(a)

(b)

Fig. 8. Cube model

signal is judged as holding a static posture temporarily, then the software reinitializes the velocity and $\mathrm{Z}$ orientation. By using filtering, tilt information, and compensation, EcoIMU can now output significantly more useful results.

\section{Time Synchronization on the Node}

Every Eco node maintains a 16-bit register that is automatically incremented by Timer 0 . The interrupt handler maintains four "unsigned int" variables (for a total of 8 bytes) as the software clock. The timer frequency is $16 \mathrm{MHz} / 4$, and therefore the minimum time unit is $0.25 \mu \mathrm{s}$. When the software clock overflows, the two Eco nodes re-synchronize to bound the time drift. The time synchronization algorithm presented here is based on a pairwise two-way message exchange [6]. Let us call the nodes $A$ and $B$.

1) Node $A$ records a timestamp $T 1$ in its local clock and sends it in a packet to Node $B$.

2) Node $B$ receives the packet and records when it receives the packet at $T 2$. Then, node $B$ records when it sends a reply packet as $T 3$. Both $T 2$ and $T 3$ are recorded in $B$ 's local clock.

3) Node $A$ receives the reply packet and records the timestamp $T 4$ in its local clock.

4) The time drift is calculated as

$$
\Delta=\frac{(T 2-T 1)-(T 4-T 3)}{2}
$$

There is also a timeout part of this algorithm. In case either the request or the reply packet is lost, node $A$ times out and retries time synchronization from the beginning.

Note that the topology consists of one server node and one or more client nodes. The client nodes are distinguished by separate IDs. In this paper, two Eco nodes are used. At the beginning, two Eco synchronize with each other and wait for two or three milliseconds, respectively, to avoid packet collision.

\section{Evaluation}

We evaluate the cube model in a series of experiments. First, we collect results from measuring synthetic motion that

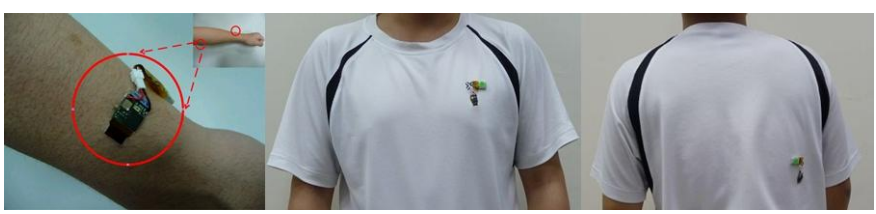

Fig. 9. Placement of Eco sensor nodes (a) lower arm (b) left front chest (c) right back chest.

includes both translation and rotation. Next, we experiment with dead reckoning (position sensing) in wearable applications involving mixed translation and rotation movements.

\section{A. Experimental Setups}

We experimented with several different geometries of EcoIMU: in a cube and on various parts of the human body. The cube enables us to validate the original mathematical model, whereas the on-body version demonstrates the practicality of the model.

1) Cube Model: We mounted two triaxial accelerometers on opposite corners of a box of the dimensions $6 \times 3.5 \times$ $5 \mathrm{~cm}^{3}$, as shown in Fig. 8. It is more rectangular than a cube model, but the equations can be easily adapted without difficulty. For convenience, we still call it a cube.

We numbered each face of this cube from 1 to 6 to better show the cube's position and orientation. The two triaxial accelerometers on the Eco nodes are oriented in the same direction, as shown in Fig. 6(b) and Fig. 8. That is, the $\mathrm{X}$ axis points rightward, the Y-axis points upward and the Z-axis points to the reader.

2) On-Body Settings: We also experimented with applying EcoIMU to different parts of the body: lower arm and upper body.

The lower arm is chosen due to its rich motions, such as waving, pounding, etc. Besides, it also represents one of the fastest moving parts of the body. One accelerometer is attached on the outside above the wrist, (Fig. 9(a)), while the other on the inside below the elbow (not shown). One can view this as further extending the cube model to an even more stretched out box, which measures $15 \times 5 \times 4 \mathrm{~cm}^{3}$.

Next, we consider the upper body as a rigid cube. One Eco node is attached on the left chest, and the other is attached on the right of the back (Fig. 9(b,c)). The relative placement is the same as the cube.

\section{B. Synthetic motion}

1) Translation: We tested translation by moving the cube to the right three times (i.e., in three separate steps with a pause between steps), to the left twice, and to the right three times, as illustrated in Fig. 10(a). The average linear velocity is $0.6 \mathrm{~m} / \mathrm{s}$, and the average linear acceleration is $9 \mathrm{~m} / \mathrm{s}^{2}$. Each step is $15 \mathrm{~cm}$ and is completed in $1 \mathrm{sec}$.

From the raw data in Fig. 11(a), one can see that the rightward motion shows up as positive-to-negative acceleration, while the leftward motion appear as negative-to-positive. The measured data do match the trend in Figs. 11(b) and 11(c). 


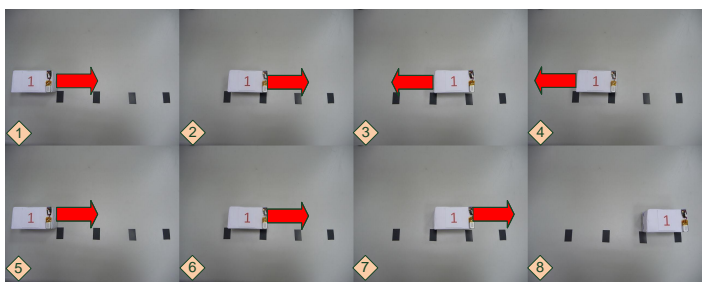

(a)

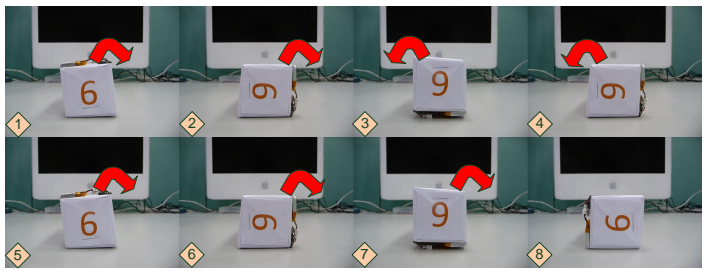

(b)

Fig. 10. Test route for cube model (a) Translation (b) Rotation

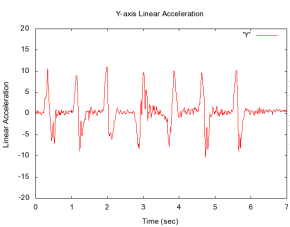

(a)

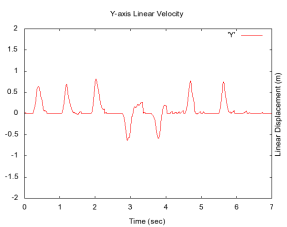

(b)

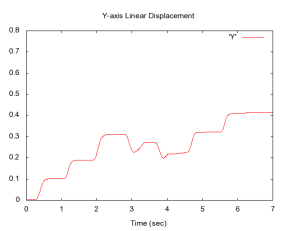

(c)
Fig. 11. Cube model translation data (a) Y-axis linear acceleration (b) Y-axis linear velocity (c) Y-axis linear displacement

After filtering, the curves of the integrated velocity and displacement are smoother and cleaner. The EcoIMU reports a displacement of $45 \mathrm{~cm}$, which is $4 \mathrm{~cm}$ from the actual displacement.

2) Rotation: We tested rotation by turning the cube upward twice (again, with a pause in between, similar to the translation test), downward twice, and upward three times. Note that Fig. 10(b) only illustrates the rotation, but the actual rotation was done in the air by hand, not on the desktop. The average angular velocity is $8 \mathrm{rad} / \mathrm{sec}$, and the average angular acceleration is $150 \mathrm{rad} / \mathrm{sec}^{2}$. Each rotation step takes 1 second, and the angle of each step is $90^{\circ}$.

Figs. 12(a) shows the angular acceleration plot. After integration, it is easier to see the angular velocity as plotted in Fig. 12(b) that corresponds to the test rotation sequence. The final angle as shown in Fig. 12(c) reaches 4.2 radians, or

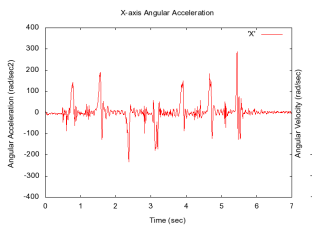

(a)

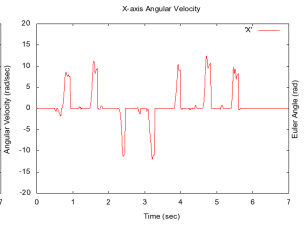

(b)

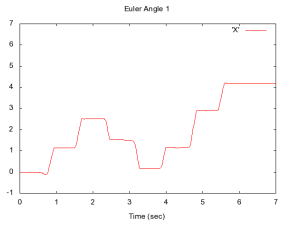

(c)
Fig. 12. Cube model rotation data (a) X-axis angular acceleration (b) X-axis angular velocity (c) $\mathrm{X}$-axis angle

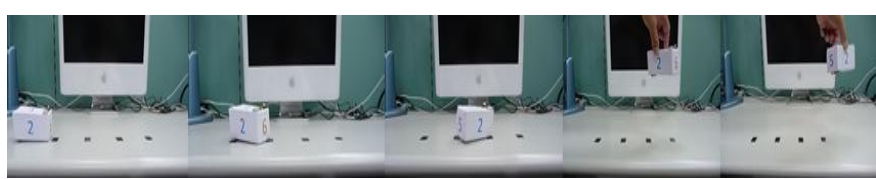

Fig. 13. Test steps for cube model dead reckoning.

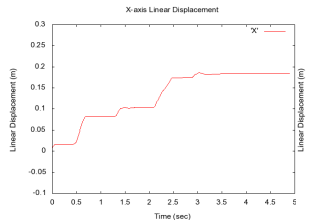

(a)

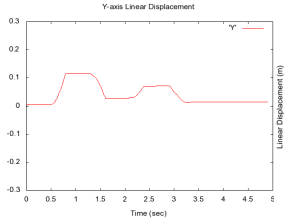

(b)

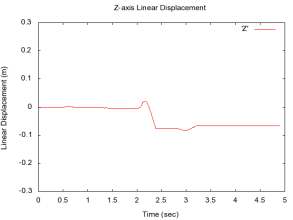

(c)

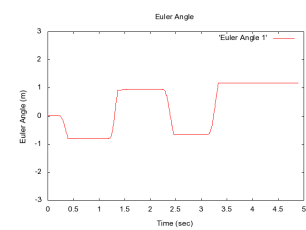

(d)

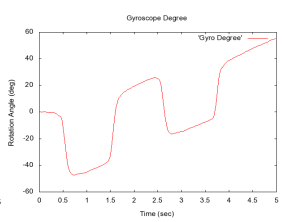

(e)
Fig. 14. Cube model dead reckoning data (a) X-axis linear displacement (b) Y-axis linear displacement (c) Z-axis linear displacement (d) X-axis Euler angle (e) Rotation degree measured by gyroscope

$240^{\circ}$, without any compensation. A significant part of the $30^{\circ}$ difference is actually due to the imprecise rotation by hand, because we did not actually put the cube on the desktop but only eyeballed the angle. Pause detection for recalibration will prove useful for compensating most of this error.

\section{Mixed Translation and Rotation}

We used EcoIMU to measure motion with mixed translation and rotation. We first tested it with the cube, followed by deployment on different parts of the body with actual human motion.

1) Cube model: Fig. 13 shows the motion sequence: we move it forward in a $60^{\circ}$ zigzag of $5 \mathrm{~cm}$ per step, lift the cube up by $10 \mathrm{~cm}$, and zigzag it further. Fig. 14(a) shows that EcoIMU measured a displacement of $20 \mathrm{~cm}$ in four stages of forward motion, which is precisely the actual displacement. Fig. 14(b) shows the Y-axis translation, which indicates the zigzag motion and is also precise. Fig. 14(c) shows the Zaxis translation, which corresponds to raising the cube. It is opposite the direction of gravity. Finally, the cube's rotation Euler angles for turning right and left $60^{\circ}$ per zigzag are also precisely measured in Fig. 14(d). It is therefore interesting to note that EcoIMU to perform very accurately in the presence of mixed rotation and translation, compared to rotation-only or translation-only types of synthetic motion.

For comparison, we used three LISY300 gyroscope modules from PARALAX. The module is a uniaxial yaw-rate sensor providing up to $300^{\circ} \mathrm{s}$ full scale rotation detection at up to $88 \mathrm{~Hz}$, and we sampled it at $50 \mathrm{~Hz}$. Due to the low sampling rate and lack of filtering and compensation, the error accumulated caused the instability in the data. Nevertheless, 
TABLE I

COMPARISON OF POWER CONSUMPTION

\begin{tabular}{|c|c|c|c|}
\hline & accelerometer & gyroscope & total \\
\hline \hline EcoIMU & $0.36 \mathrm{~mA} \times 3 \mathrm{~V} \times \mathbf{2}$ & none & $2.16 \mathrm{~mW}$ \\
\hline gyro IMU & $0.36 \mathrm{~mA} \times 3 \mathrm{~V}$ & $5.2 \mathrm{~mA} \times 3.3 \mathrm{~V} \times \mathbf{3}$ & $52.56 \mathrm{~mW}$ \\
\hline
\end{tabular}

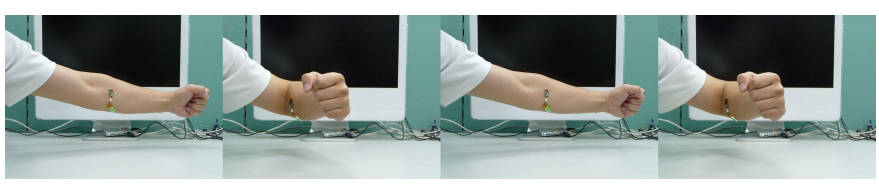

Fig. 15. Test action for lower arm.

we can still see the similar curve in Fig. 14(d) and Fig. 14(e). Besides, the power consumption of the gyroscopes is much higher than that of accelerometers. As shown in Table I, the normal IMU composed of one triaxial accelerometer and three uniaxial gyroscopes costs $52.56 \mathrm{~mW}$, which is 24 times that of EcoIMU. This result shows the advantages of EcoIMU in terms of accuracy and low power consumption.

2) On-body Deployment: As mentioned in Section V-A2, we experimented with deployment on the lower arm and the upper body. The experimental results are as follows.

To test EcoIMU on the forearm, the test subject (one of the authors) first holds the forearm straight out, rotates it $90^{\circ}$ back to be parallel to the chest, and repeats these two steps, as shown in Fig. 15. That is, the X-Z plane is rotated about the $\mathrm{Y}$ axis by $90^{\circ}$. Because EcoIMU measures the displacement of the half-way point between these two accelerometer, the rotation radius is from the elbow to the point which is $15 \mathrm{~cm}$, and thus the actual displacement is $1 / 2 \times \pi \times 15 \mathrm{~cm}=$ $23.55 \mathrm{~cm}$. The $\mathrm{X}$ and $\mathrm{Z}$ displacements as measured vary from $18 \mathrm{~cm}$ to $25 \mathrm{~cm}$ as shown in Figs. 16(a) and 16(b). However, the rotation angles are surprisingly accurate. Fig. 16(c) shows

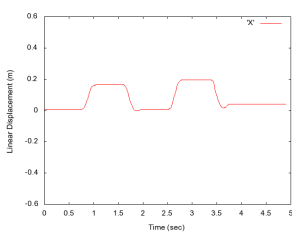

(a)

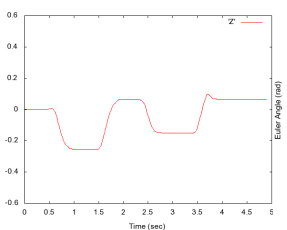

(b)

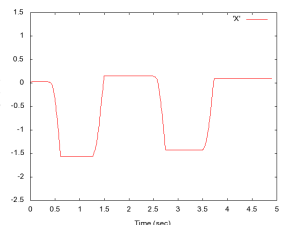

(c)
Fig. 16. Lower arm data (a) X-axis linear displacement (b) Z-axis linear displacement (c) X-axis Euler angle

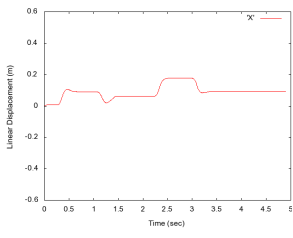

(a)

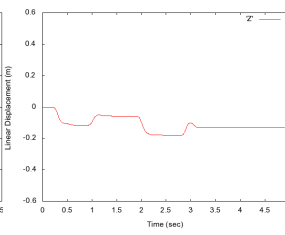

(b)

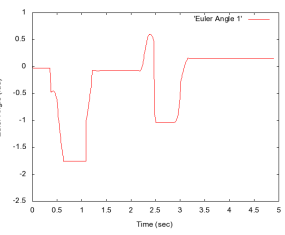

(c)
Fig. 17. Upper body data. (a) X-axis linear displacement (b) Z-axis linear displacement (c) X-axis Euler angle. the measured rotation angle to be 1.5 radians, or $86^{\circ}$, which is very close to $90^{\circ}$.

For the upper body test, the test subject twists his body to the right and back about his waist twice without moving his lower body. He attempts to keep the two accelerometers as close to fixed relative positions as possible. The actual Xaxis translation is $15 \mathrm{~cm}$ on average per step, and Fig. 17(a) shows the final error to be about $5 \mathrm{~cm}$ in the $\mathrm{X}$ translation, and Fig. 17(b) shows $10 \mathrm{~cm}$ error in the $\mathrm{Z}$ translation. On the other hand, for rotation, Fig. 17(c) shows that the first two steps are accurate as expected $\left(90^{\circ}\right)$, but the last two steps are off by $10-20^{\circ}$. The inaccuracy is primarily due to the fact that the human body is not rigid. The problem is further complicated by loose clothing, which introduces additional noise beyond what can be filtered or compensated. It would work much better if the two accelerometers were deployed on a rigid armor or a helmet, but they would be less convenient.

\section{CONCLUSIONS}

EcoIMU, is the first practical implementation of the gyrofree IMU idea based on a pair of triaxial accelerometers. It expands the application of accelerometers to absolute motion tracking, not just relative motion tracking. The use of accelerometers significantly reduces the power and cost of the IMU. We overcame several limitations with accelerometerbased systems by leveraging domain knowledge, such as static posture detection and recalibration. We demonstrated the feasibility with an actual implementation in the form of a wireless body sensor system. The compact form factor and the flexibility in geometry makes it possible to choose many different parts of the body for deployment, depending on what is convenient to the user. Future work includes moving more computation to the mobile unit without depending on a powerful PC to perform the backend calculation.

Acknowledgments: The authors thank Guo-Huei Chang and Shun-Yao Yang with gyroscopes. This work was sponsored by the National Science Foundation CNS-0448668, CNS0721926, CBET-0933694, the National Science Council (Taiwan) NSC 96-2218-E-007-009, and Ministry of Economy (Taiwan) 98-EC-17-A-07-S1-001.

\section{REFERENCES}

[1] A. Padgaonkar, K. Krieger, and A. King, "Measurement of angular acceleration of a rigid body using linear accelerometers," Journal of Applied Mechanics, Transactions of the American Society of Mechanical Engineers, vol. 42, pp. 552-556, September 1975.

[2] J.-H. Chen, S.-C. Lee, and D. B. DeBra, "Gyroscope free strapdown inertial measurement unit by six linear accelerometers," Journal of Guidance, Control, and Dynamics, vol. 17, no. 2, March-April 1994.

[3] C. Tan, S. Park, K. Mostov, and P. Varaiya, "Design of gyroscope-free navigation systems," IEEE Intelligent Transportation Systems Conference Proceedings, August 2001.

[4] S. Park, C.-W. Tan, and J. Park, "A scheme for improving the performance of a gyroscope-free inertial measurement unit," Sensors and Actuators A: Physical, vol. 121, no. 2, pp. 410-420, March 2005.

[5] K. Parsa, J. Angeles, and A. K. Misra, "Rigid-body pose and twist estimation using an accelerometer array," Archive of Applied Mechanics, vol. 74, pp. 223-236, December 2004.

[6] S. Ganeriwal, R. Kumar, and M. B. Srivastava, "Timing-sync protocol for sensor networks," The ACM Conference on Embedded Networked Sensor Systems, pp. 138-149, November 2003. 